\title{
Viabilidad de propuestas presidenciales por parte de candidatos que aspiran al poder ejecutivo en Colombia
}

\section{Feasibility of presidential proposals by candidates who aspire to the executive power in Colombia}

Juan Arévalo Téllez, Adrián Barrero Buitrago, Nidia Castañeda Barroso, Andrés Erazo Pazos y Juan Figueroa Pico*

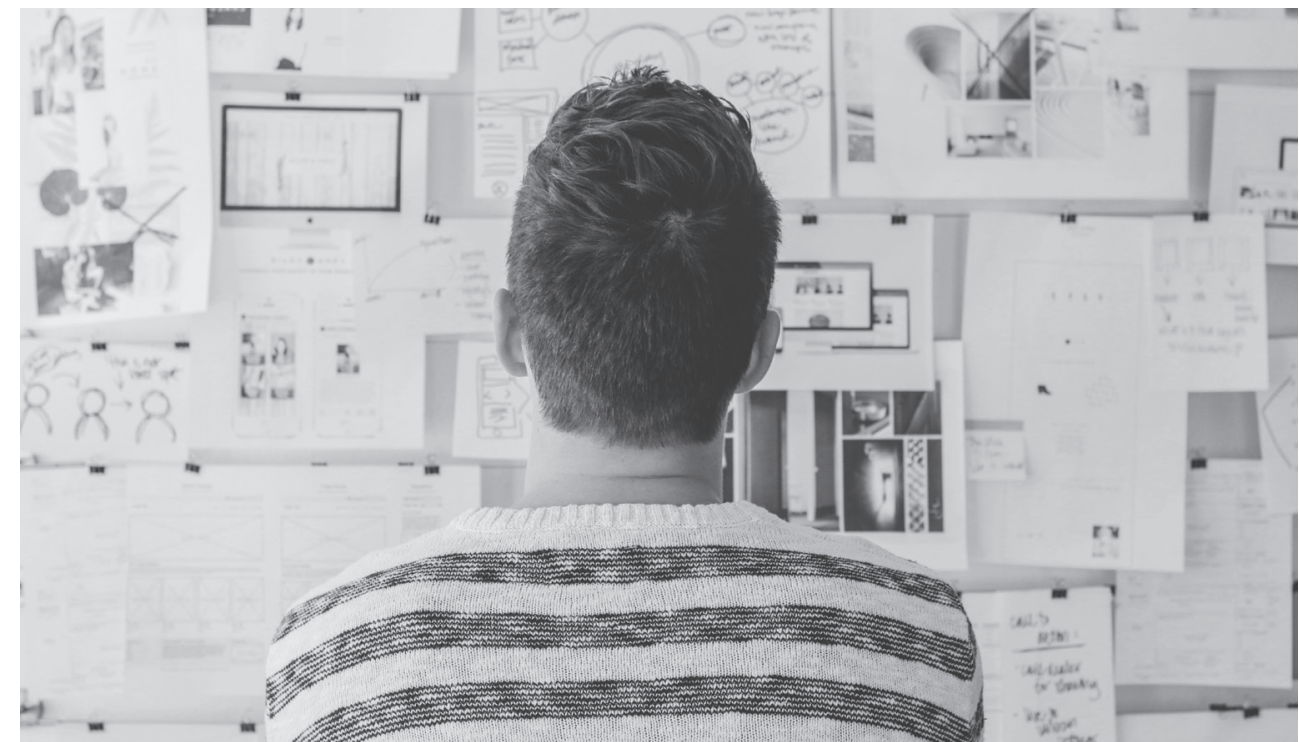

\section{Resumen}

La presente investigación lleva a cabo un estudio riguroso de cada plan presidencial de los candidatos que aspiran al poder ejecutivo en el año 2018. De acuerdo con esto, se pretende plasmar qué tan viable es lo que propone cada aspirante a la presidencia con el fin de dar un juicio concreto de lo que es más beneficioso para el país en términos económicos, de lucha contra la corrupción y educación. Este estudio está en función de la participación de encuestados (en particular profesores de la Universidad Nacional

Citar este artículo como: Arévalo Téllez, J., Barrero Buitrago, A., Castañeda Barroso, N., Erazo Pazos, A. y Figueroa Pico, J. (2018). Viabilidad de propuestas presidenciales por parte de candidatos que aspiran al poder ejecutivo en Colombia. Revista Papeles, 10(19), 48-55.

Fecha de recibido: 22 de febrero de 2018

Fecha de aceptación: 20 de mayo de 2018

* Estudiantes de Economía de la Universidad Nacional de Colombia. Trabajo realizado bajo la dirección del profesor Enrique Ferrer-Corredor, en la cátedra de Metodología de la investigación. Correos electrónicos: jarevalot@unal.edu.co, abarrero@unal.edu.co,nicastanedab@unal.edu.co, aerazo@unal.edu.co, jfigueroap@ unal.edu.co 
de Colombia, sede Bogotá, de la escuela de Ciencias Económicas y Facultad de Ciencias Sociales). Por otra parte, se tienen en cuenta cuatro variables importantes para el análisis de los planes presidenciales por parte de los encuestados (viable, inviable, conoce el plan de los demás candidatos, no conoce el plan de los demás candidatos) para lograr abarcar toda la información más adelante presentada y sustentada.

Palabras clave: poder ejecutivo, propuestas presidenciales, viabilidad, inviabilidad, encuestas, profesores.

\begin{abstract}
The present investigation carries out a rigorous study of each presidential plan of the candidates that aspire to the executive power in the year 2018. In accordance with this, it is aimed to capture how viable is what each candidate for the presidency proposes, in order to give a concrete judgment of what it is more beneficial for the country in economic terms, fight against corruption and education. This study is based on the participation of a particular public (professors of the National University of Colombia, Bogotá campus, of the Faculties of Economic and Social Sciences). On the other hand, four important variables are taken into account for the analysis of the presidential plans by the respondents (viable, unfeasible, knows the candidate's plan, does not know the candidate's plan) to achieve covering all the information later presented and supported.
\end{abstract}

Keywords: executive power, presidential proposals, viability, unfeasibility, survey, professors.

\title{
Introducción
}

La tendencia que los colombianos han tenido al momento de elegir a su agente representativo en una democracia, haciendo alusión al poder ejecutivo, ha tenido la mayoría de veces un impacto negativo, pues no analizan con detalle cada propuesta del respectivo candidato presidencial para así elegirlo con criterio de autoridad $-\mathrm{y}$ previo conocimiento- de la manera más eficaz. Sin embargo, han existido perspectivas político-ideológicas que inculcan miedo a la población colombiana, con lo cual se distorsiona la elección del ciudadano. Además, la cultura colombiana, debido al crecimiento histórico que ha tenido a lo largo de los años, ha estado arraigada a sesgos ideológicos (orientación distorsionada del entorno, en este caso entorno político-democrático) que desarrollan una capacidad de percepción e interpretación muy distorsionada al momento de tomar decisiones que, de cierta manera, poseen una implicación de gran envergadura para el futuro del individuo decisor; y no solo de él, sino también del futuro de las personas en su entorno. Por tal razón, es conveniente interpretar este factor en función de la manera en que las personas eligen a quien obtendrá el poder ejecutivo por los próximos cuatro años y, también, cómo este tipo de decisiones afectan el bienestar a largo plazo de las personas.

Además, es importante saber que la postura ideológica de las personas (actitud que posee el individuo decisor, y que en este caso puede ser de tres tipos: izquierda, centro, derecha) influye bastante en el modo en que toman sus decisiones de elección. En consecuencia, la cognición social que se tiene con cada persona desata distintos niveles de intercambio en la decisión; por tal razón, la manera en como 
el discurso afecta los sentidos y, por lo tanto, la forma en que se percibe la realidad de las personas es un factor crítico y determinante del sesgo que pueden imponer las externalidades dadas en el entorno: partidos políticos, noticias, radio, televisión, candidatos, figuras públicas, etc. (van Dijk, 1994).

Por tal motivo, las elecciones presidenciales en Colombia, la mayoría de veces, han estado influenciadas por este factor negativo en la sociedad, debido a que los medios masivos de transferencia de información al público son manejados por ciertas minorías que buscan en la mayoría de casos un bienestar particular. Es por eso que esta investigación intenta decir cuál es la manera más óptima y eficaz de entender la problemática sociocultural de nuestro país en un entorno de elecciones presidenciales.

Debido a lo anterior, y gracias a que el modelo de estudio de esta realidad es bastante complejo de comprender si no se simplifica de manera adecuada el entorno de estudio, en esta investigación se reduce el análisis en cómo los individuos toman sus decisiones, enfocándose solamente en un reducido público académico, es decir, profesores de la Facultad de Ciencias Sociales y Escuela de Economía de la Universidad Nacional de Colombia, sede Bogotá.

De esta manera, nuestro análisis demuestra cómo es el patrón de decisión de cada individuo y cómo influye el sesgo ideológico en la toma de estas decisiones. Igualmente, esta simplificación tiene en cuenta cuatro variables de estudio en función de los planes presidenciales que están sustentados por medio de encuestas. Finalmente, con la ejecución de esta metodología, argumentamos cuáles propuestas presidenciales son más viables y cuáles pueden ser perjudiciales para el país.

\section{Metodología de la investigación}

Para iniciar el análisis de esta investigación se describe el proceso de recolección y la posterior evaluación de datos, para encontrar el aspecto central y crítico en la evaluación de toma de decisiones. En primera instancia, se utiliza una metodología que hace uso de encuestas dirigidas al público académico (profesores de la Facultad de Ciencias Sociales y Escuela de Economía), en donde se plasman las propuestas de los candidatos aspirantes a la presidencia en Colombia. Los factores tenidos en cuenta y plasmados en las encuestas están dados en términos de: economía del país, educación y lucha contra la corrupción.

Así mismo, el estudio busca identificar el patrón de respuesta, es decir, si hay relación de sesgos ideológicos o no. Por tal motivo, la estructura de las encuestas está clasificada en dos momentos: a. Cuando saben el nombre del candidato, es decir, el encuestado conoce de quién es la propuesta presidencial.

b. Cuando no sabe el nombre del encuestado, es decir, describe a detalle la propuesta del candidato; no obstante, el encuestado no sabe quién la propone.

Del mismo modo, las encuestas están en función de clasificar tales propuestas, de manera que se emplea un rango de elección sencillo y eficaz: no viable (1-2), no sabe-no responde (3), viable (4-5). Así pues, el encuestado, al responder, nos da la información para cuantificar la viabilidad de las propuestas e interpretar el sesgo ideológico que existe (debido a que algunas propuestas no son explícitas con el nombre del candidato que las sustenta).

A continuación se presenta la ficha técnica de la encuesta, para una mayor comprensión del proceso de recolección de datos. 
Tabla 1. Ficha técnica de recolección de datos

\begin{tabular}{l|l|l|l|} 
Universo & $\begin{array}{l}\text { Profesores de la Facultad de Ciencias Sociales y Escuela de Economía de la sede } \\
\text { Bogotá } \\
\text { Unidad de } \\
\text { muestreo }\end{array}$ & $\begin{array}{l}40 \text { profesores de la Escuela de Economía }(70 \%) \text { y de la Facultad de Ciencias Sociales } \\
(30 \%)\end{array}$ \\
$\begin{array}{l}\text { Fecha } \\
\text { Entre el } 22 \text { de febrero y el } 16 \text { de mayo del } 2018\end{array}$ \\
$\begin{array}{l}\text { Delimitación } \\
\text { geográfica }\end{array}$ & Universidad Nacional de Colombia (Bogotá D. C.) \\
$\begin{array}{l}\text { Objetivos de } \\
\text { la encuesta }\end{array}$ & $\begin{array}{l}\text { Exponer la viabilidad de las propuestas presidenciales, explicar el sesgo que existe al } \\
\text { momento de decidir el candidato correcto o de preferencia y sustentar las propuestas } \\
\text { más viables al modelo de desarrollo del país. }\end{array}$ \\
\hline $\begin{array}{l}\text { Número de } \\
\text { preguntas }\end{array}$ & $\begin{array}{l}\text { En este apartado es importante aclarar que la encuesta está diseñada con } 15 \text { pregun- } \\
\text { tas relacionadas a los planes presidenciales, además de una única pregunta (que es la } \\
\text { intención de voto); es decir, } 5 \text { opciones de respuesta (los candidatos presidenciales de } \\
\text { este estudio). }\end{array}$ \\
\hline
\end{tabular}

Fuente: elaborada por los autores

\section{Encuestas}

La información plasmada en las encuestas está sustentada con base a los planes presidenciales que cada candidato ha compartido públicamente por los medios masivos de comunicación. De manera que en este estudio se tuvieron en cuenta las propuestas relacionadas con temas de economía, educación y lucha contra la corrupción, debido a que estos han sido de gran controversia a lo largo de cada elección presidencial y también en los debates presidenciales efectuados en el año 2018. Por añadidura, se tuvieron en cuenta 3 propuestas por cada candidato, que en este estudio son 5 : Germán Vargas Lleras, Iván Duque, Humberto de la Calle, Sergio Fajardo y Gustavo Petro.
De la misma manera, la metodología empleada en las encuestas está estructurada del siguiente modo: hay 8 preguntas aleatorias en las que no se sabe quién es el autor de la propuesta y 7 preguntas en las que sí se sabe exactamente el autor de la propuesta; por consiguiente, existe la forma de cuantificar si existe un sesgo al momento de elección. Incluso, estructuramos un rango de 1 a 5 (1-2: no viable, 3: no sabe no responde, 4-5: viable) con el objetivo de encontrar qué propuestas son viables y cuáles no, es decir que cada propuesta tiene un rango de elección en donde el encuestado decide.

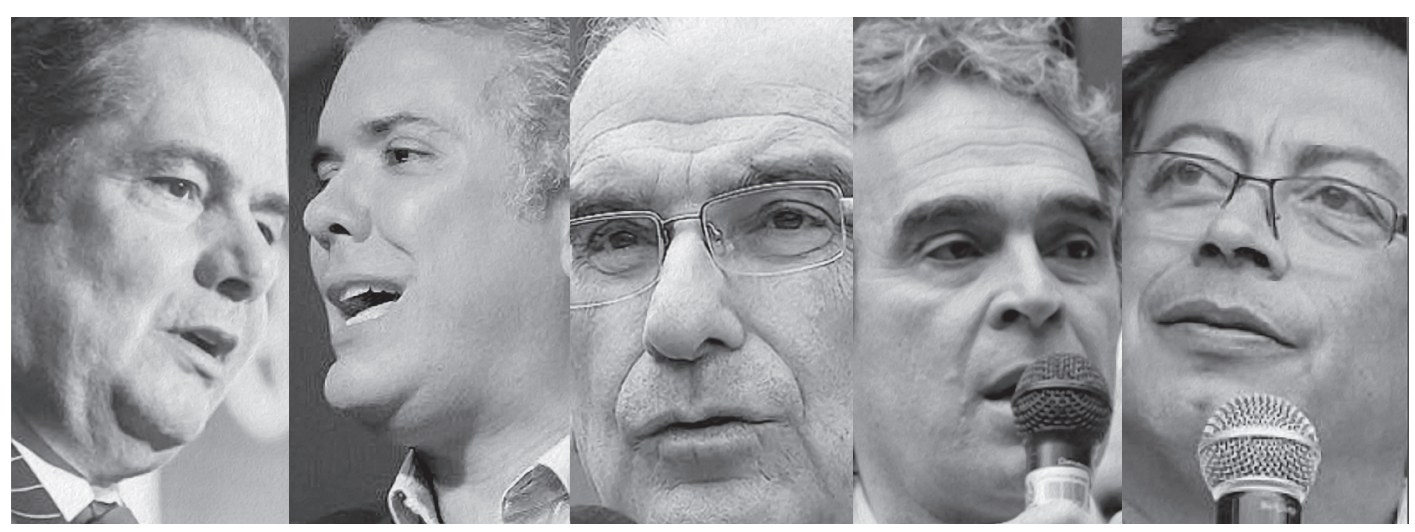

Revista PAPELES • ISSN 0123-0670 • Vol. 10(19) • pp. 48-55 • Enero-junio de 2018 


\section{Matriz}

La siguiente matriz (tabla 2) detalla la manera en que se ejecutó la recolección y cuantificación de los datos obtenidos por medio de las encuestas ${ }^{1}$.

Es importante mencionar que el espacio muestral es de 40 encuestados y que por cada encuesta se realizaron 15 preguntas. Asimismo, cada número en el recuadro respectivo a cada candidato concierne a la respuesta seleccionada por el encuestado. Por último, en la tabla 3 detallamos el patrón de respuesta de intención de voto.

Tabla 2. Matriz de recolección de respuestas

\begin{tabular}{l|c|c|c|c|c|c|c|c|c|c|}
\hline \multicolumn{19}{c|}{ Vo Sabe-No Responde: 86} \\
\hline & Petro & Fajardo & De la Calle & Lleras & Duque & Petro & Fajardo & De la Calle & Lleras & Duque \\
\hline Sabe & 27 & 40 & 15 & 17 & 20 & 19 & 18 & 23 & 22 & 36 \\
\hline $\begin{array}{l}\text { No sabe } \\
\text { Total encuestados: }\end{array}$ & 26 & 19 & 37 & 17 & 26 & 28 & 26 & 27 & 47 & 24 \\
\hline
\end{tabular}

Fuente: elaborada por los autores

Tabla 3. Respuesta única de intención de voto

\begin{tabular}{|c|c|c|c|c|}
\hline Petro & Fajardo & Lleras & Duque & De la Calle \\
\hline 9 & 14 & 1 & --- & 16 \\
\hline encuestados & & 40 & & \\
\hline
\end{tabular}

Total encuestados

Fuente: elaborada por los autores

\section{Análisis de datos}

A continuación se presenta la información cuantificada de las encuestas realizadas a 40 profesores de la Facultad de Ciencias Sociales y Escuela de Economía de la Universidad Nacional de Colombia (sede Bogotá).

En la gráfica 1 se presenta un resumen de la investigación, en donde se muestra en porcentajes cómo los encuestados responden cuando

1 Puede encontrarse el material dirigiéndose a: https://drive.google.com/file/d/1LzH1wJ7Qw2GS 0zGdMNLvqQPUkg54zhXf/view?usp=sharing

2 Cada número en el cuadro correspondiente a cada candidato hace referencia al número de veces que los encuestados eligieron la respectiva propuesta; un total de 640 respuestas.
Gráfica 1. Resumen de encuestas

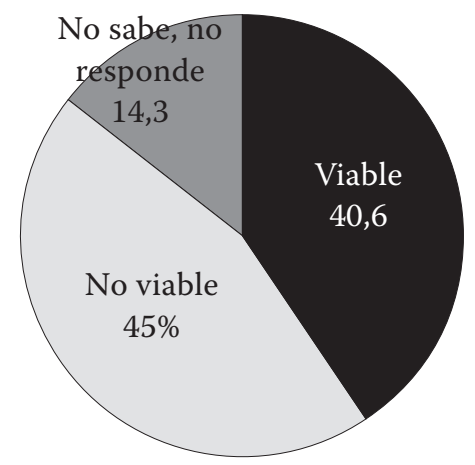

Número total de repuestas: 600

Fuente: elaborada por los autores 
Gráfica 2. Propuestas viables (explícita)

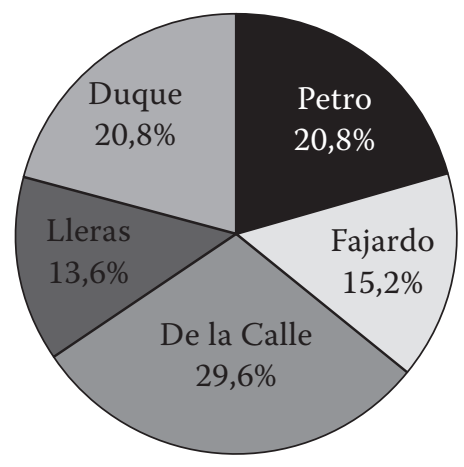

Número total de respuestas: 125

Fuente: elaboradas por los autores

Gráfica 3. Propuestas viables (implícita)

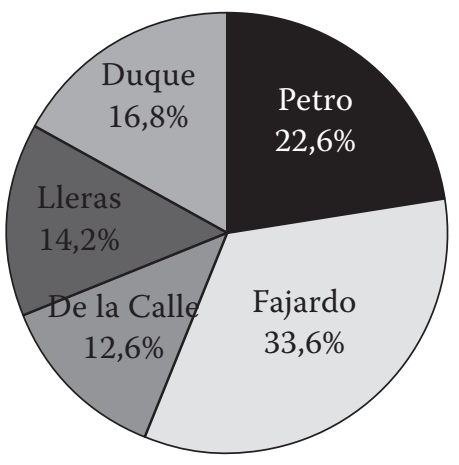

Número total de respuestas: 119

Fuente: elaboradas por los autores

leen las propuestas presidenciales. Esto en un panorama general, después presentamos de manera detallada cada porcentaje. Iniciamos con las propuestas viables.

En la gráfica 2 se detalla la viabilidad de las propuestas de cada candidato. Es importante mencionar que en este apartado los encuestados saben quién es el autor de la propuesta. Observamos que el candidato Sergio Fajardo es el más viable con sus propuestas en términos de economía, educación y lucha contra la corrupción.

Sin embargo, en la gráfica 3 se detalla también la viabilidad de las propuestas de cada candidato; no obstante, en este apartado la metodología de la encuesta cambió, debido a que ahora los encuestados no saben quién es exactamente el autor de la propuesta, por lo cual observamos el cambio de decisión por parte del encuestado. Sin embargo, el porcentaje obtenido por Humberto de la Calle (gráfica 3) es menor que el de Sergio Fajardo (gráfica 2).

De acuerdo con lo anterior, se observa la tendencia que existe de cambio de elección cuando se presentan 2 escenarios (conoce, incógnito) en un entorno de elecciones presidenciales. Van Dijk (1994) explica que la forma en que el lector interpreta un texto se debe a las relaciones semánticas que este representa, pero fundamentalmente a la estrategia que el lector emplea de acuerdo a las hipótesis y expectativas generadas; de aquí se deduce que el encuestado decide de acuerdo con sus expectativas. Pero además hay un claro sesgo de elección, porque los autores de este estudio, al momento de realizar las encuestas, observamos una clara acción de rechazo ante cierto nombre de algunos de los candidatos, es decir, el encuestado no se tomaba la tarea de leer las propuestas, sino que al momento de observar el nombre del autor inmediatamente marcaba viable, inviable o, en su defecto, no sabe o no responde.

A continuación, presentamos la misma metodología pero con las propuestas inviables.

Gráfica 4. Propuestas inviables (explícita)

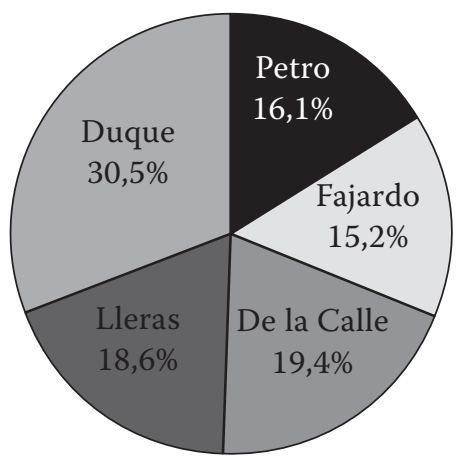

Número total de respuestas: 118

Fuente: elaboradas por los autores 
Gráfica 5. Propuestas inviables (implícita)

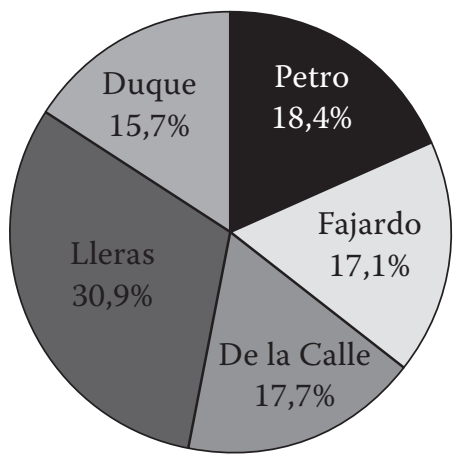

Número total de respuestas: 152

Fuente: elaboradas por los autores

En la gráfica 4 se presenta la información recolectada en función de los candidatos que tienen las propuestas menos viables en términos de economía, educación y lucha contra la corrupción para el país; además, en este apartado los encuestados saben quién es el autor de las propuestas planteadas. Así pues, se detalla que las propuestas menos viables son las del candidato Iván Duque, con un 30,5 $\%$ de votación.

Después, en la gráfica 5 , se presenta la información pero con la condición de que ahora los encuestados no saben quién es el autor de la propuesta presidencial. Se observa que ahora ellos son más sensibles a elegir las propuestas del candidato Germán Vargas Lleras, calificándolas como las más inviables, con un porcentaje de 30,9 \%; y ahora el candidato con el índice más bajo de inviabilidad es Iván Duque, con un porcentaje del 15,7 \%; de manera que se observa el modo en que los individuos deciden de acuerdo a la interpretación explícita que existe en el enunciado de las encuestas.

\section{Conclusiones}

Este estudio encontró que la manera en que los individuos eligen su candidato no está cien por cien sustentada por las propuestas que este propone desarrollar en su periodo de gobierno en el país.
De acuerdo con lo anterior, esta investigación encontró que la manera en que algunos individuos toman decisiones está en relación con la manera que se presenta explícitamente la información y, ligado a esto, los sesgos ideológicos están claramente presentes (esto según la experiencia de los autores de esta investigación, quienes evidenciaron cómo las respuestas de los encuestados diferían en su forma de elección solamente con mirar el nombre del candidato propuesto). Además, es importante mencionar que, según van Dijk (1994), la actitud que asume el individuo está en función de estructuras y principios que son base de la ideología del sujeto; por esto, la manera en que los encuestados respondieron arroja tales resultados.

Finalmente, se presenta la manera en que los encuestados respondieron a su intención de voto, la cual es sencilla y eficaz, es decir, una sola pregunta y cinco formas de elección (los candidatos que están en este estudio). Para una mejor comprensión de la cuantificación de los datos plasmados a continuación, esta información está disponible en la matriz de recolección de datos antes mencionada.

Gráfica 6. Intención de voto

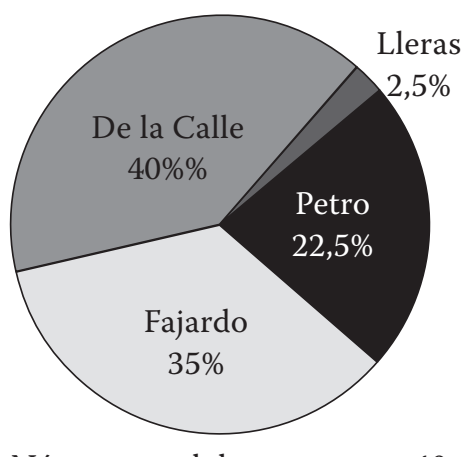

Número total de respuestas: 40

Fuente: elaboración de los autores
Desde una perspectiva normativista, la manera de elección no está sujeta a la comprensión y correcta elección de lo más viable en términos de educación, economía y lucha contra la corrupción hacia el país, sino que, de cierta 
manera, hay un sesgo ideológico que impide la optimización de las decisiones que se toman en un entorno de elecciones presidenciales. Así mismo, hubo una tendencia positivista cuando se analizó y recolectó la información en este estudio, es decir, hay una clara diferencia de elección de candidato cuando se conoce al autor de las propuestas; por lo que se deduce que el espacio de estudio, con respecto a la elección de candidatos, no está en función de lo más idóneo y correcto para el país, ya que la manera correcta en que cada sujeto lo analiza subjetivamente es lo que se escoge para el futuro del mismo.

De acuerdo con las gráficas 2 y 3 observamos el cambio de respuesta que los encuestados tuvieron; es decir, primero eligieron las propuestas y las clasificaron como las más viables con respecto al candidato Fajardo (aquí conocían el nombre del autor de la propuesta). Pero cuando se cambió la metodología de la encuesta (ya no se explicitaba quién proponía el plan de gobierno), los encuestados estuvieron de acuerdo con que las propuestas más viables las tenía Humberto de la Calle. En otras palabras, no hay una forma objetiva y neutral de elegir al candidato, es decir, del modo en que realmente debería ser (normativa), sino que existe un prejuicio que, de cierta manera, está fundamentado por ideologías culturales, sociales, que han sido inculcadas por medios masivos, familiares, conocidos, etc.; por lo cual, en este tipo de ambientes, la forma en que realmente se manifiesta (positivista) la decisión de los candidatos presidenciales está basada en lo antes mencionado. Por otro lado, las gráficas 4 y 5 están sustentadas de la misma manera, pero en función de propuestas inviables, es decir, los encuestados escogían al candidato que proponía lo menos apto para el país en términos de economía, educación y lucha contra la corrupción.

Incluso en las gráficas 4 y 5 se observa que Iván Duque y Germán Vargas Lleras poseen las propuestas menos viables para el país, respectivamente. Iván Duque está en el marco de: "cuando saben el nombre del autor de las propuesta" y, por otra parte, German Vargas Lleras está en el marco de: "cuando no saben el nombre del autor de la propuesta".

Finalmente, en la gráfica 6 se presenta la intención de voto de los encuestados. Este estudio encontró que Humberto de la Calle y Sergio Fajardo son los candidatos de preferencia de los profesores encuestados de la Facultad de Ciencias Sociales y Escuela de Economía de la Universidad Nacional de Colombia (sede Bogotá). Por añadidura, y en contraste con la elección de propuestas viables, hay un acierto con los candidatos antes mencionados, es decir, los profesores eligieron como candidatos con las propuestas más viables a Humberto de la Calle y Sergio Fajardo, por lo cual este estudio demuestra que a criterio de los académicos encuestados (40), dichos candidatos son los más idóneos para el país en relación con temas como: economía, educación y lucha contra la corrupción.

Por último, la realidad que se presenta con respecto a la toma de decisiones en un escenario de elecciones presidenciales sigue arraigada a sesgos ideológicos; no obstante, la manera de elección de los académicos de la Universidad Nacional (sede Bogotá) tiene la característica de ser crítica en términos de: economía, lucha contra la corrupción y educación ${ }^{1}$.

\section{Referencias}

Van Dijk, T. (1994). Discurso, Poder y Cognición Social. En Cuadernos N. 2. doi: https:// doi.org/10.4067/S0718-237620050001000

\footnotetext{
Se hace alusión a que las decisiones se caracterizan por ser críticas debido al uso de una elección neutral del respectivo candidato, es decir, sin importar el nombre del autor de la propuesta presidencial los encuestados leyeron y eligieron con detenimiento y análisis crítico (esto también argumentado con la experiencia de los autores de este estudio al observar los distintos comportamientos de cada encuestado).
} 